\title{
Age trends in 30 day hospital readmissions: US national retrospective analysis
}

\author{
Jay G Berry, ${ }^{1,2}$ James C Gay, ${ }^{3}$ Karen Joynt Maddox, ${ }^{4}$ Eric A Coleman, ${ }^{5}$ Emily M Bucholz, ${ }^{1,2}$ \\ Margaret R O’Neill, ${ }^{1}$ Kevin Blaine, ${ }^{1}$ Matthew Hall ${ }^{6}$
}

${ }^{1}$ Division of General Pediatrics,

Boston Children's Hospital,

Boston, MA 02115, USA

${ }^{2}$ Department of Pediatrics,

Harvard Medical School,

Boston, MA 02115, USA

${ }^{3}$ Monroe Carell Jr Children's

Hospital at Vanderbilt

Department of Pediatrics,

Vanderbilt University Medical

Center, Nashville, TN 37232,

USA

${ }^{4}$ Washington University School

of Medicine, St Louis, MO

63110, USA

${ }^{5}$ Division of Health Care Policy and Research, University of Colorado School of Medicine,

Aurora, CO 80045, USA

${ }^{6}$ Children's Hospital

Association, Lenexa, KS 66219, USA

Correspondence to: J G Berry Jay.berry@childrens.harvard.edu

Cite this as: BMJ 2018;360:k497 http://dx.doi.org/10.1136/bmj.k497

Accepted: 15 January 2018

\section{ABSTRACT}

OBJECTIVE

To assess trends in and risk factors for readmission to hospital across the age continuum.

DESIGN

Retrospective analysis.

SETTING AND PARTICIPANTS

31729762 index hospital admissions for all conditions in 2013 from the US Agency for Healthcare Research and Quality Nationwide Readmissions Database.

\section{MAIN OUTCOME MEASURE}

30 day, all cause, unplanned hospital readmissions. Odds of readmission were compared by patients' age in one year epochs with logistic regression, accounting for sex, payer, length of stay, discharge disposition, number of chronic conditions, reason for and severity of admission, and data clustering by hospital. The middle ( 45 years) of the age range (0$90+$ years) was selected as the age reference group. RESULTS

The 30 day unplanned readmission rate following all US index admissions was $11.6 \%(n=3678018)$. Referenced by patients aged 45 years, the adjusted odds ratio for readmission increased between ages 16 and 20 years (from 0.70 (95\% confidence interval 0.68 to 0.71 ) to 1.04 (1.02 to 1.06)), remained elevated between ages 21 and 44 years (range 1.02 (1.00 to 1.03 ) to 1.12 (1.10 to 1.14$)$ ), steadily decreased between ages 46 and 64 years (range 1.02 (1.00 to 1.04$)$ to 0.91 (0.90 to 0.93)), and decreased abruptly at age 65 years (0.78 (0.77 to 0.79$))$, after which the odds remained relatively constant with advancing age. Across all ages, multiple chronic

\section{WHAT IS ALREADY KNOWN ON THIS TOPIC}

Hospital readmissions are important to clinical leaders and policy makers worldwide, as reducing these events can improve care and reduce costs In contrast to other countries, readmission policy, as well as clinical interventions to reduce readmissions in the US, has largely focused on people aged 65 years and older

Little is known about which individuals, by age, have the highest odds of readmission

\section{WHAT THIS STUDY ADDS}

Accounting for demographic and clinical risk, the adjusted odds of readmission increased during adolescents' transition to adulthood, peaked by middle age, and decreased at age 65 years

For children and adults, multiple chronic conditions and index admissions for a mental health problem were strongly correlated with readmission Further attention to the measurement, causes, and opportunities for reduction of readmissions in adolescents as well as young and middle aged adults is warranted

conditions were associated with the highest adjusted odds of readmission (for example, 3.67 (3.64 to 3.69) for six or more versus no chronic conditions). Among children, young adults, and middle aged adults, mental health was one of the most common reasons for index admissions that had high adjusted readmission rates ( $\geq 75$ th centile).

\section{CONCLUSIONS}

The likelihood of readmission was elevated for children transitioning to adulthood, children and younger adults with mental health disorders, and patients of all ages with multiple chronic conditions. Further attention to the measurement and causes of readmission and opportunities for its reduction in these groups is warranted.

\section{Introduction}

Hospital readmissions are important to patients, families, clinicians, and policy makers throughout the world, as reducing these events can improve care and reduce costs. Healthcare policies to reduce readmissions have been deployed in several countries, including Denmark, England, Germany, and the United States. ${ }^{1}$ Thus far, readmission policy, as well as clinical interventions to reduce readmissions, in the US has largely focused on the over 65, fee for service Medicare population, which is the target of the Centers for Medicare and Medicaid Services' Hospital Readmission Reduction Program. ${ }^{2}$ However, in the US and elsewhere, health plans, states, federal agencies, and others are increasingly committing to broad, population based strategies that will optimize the care transitions and the health of all people, regardless of their age. ${ }^{3}$

Understanding which people, by age, have the greatest odds of readmission could help to direct the assessment and development of new opportunities to focus on hospital readmissions beyond the elderly population. Existing national reports from the Agency for Healthcare Quality and Research on hospital readmissions for all US people suggest that readmission rates vary substantially across the age range. ${ }^{4-8}$ For example, unadjusted 30 day hospital readmission rates are much lower in children than in older adults. ${ }^{56}$ Lower readmission rates for children compared with older people have also been reported in England. ${ }^{9}$ Although helpful in advancing knowledge about readmissions across the age continuum, the US reports in particular are limited by not excluding elective readmissions or adjusting for prominent demographic and clinical risk factors, including payer and number of chronic conditions. ${ }^{10-14}$ US readmission studies on condition specific admissions for adults only (that is, excluding children) that include methods 
to account for these factors suggest that younger adults may have odds of readmission that are comparable to or higher than those of older people. ${ }^{15} 16$ Several studies in developing countries (such as Hong Kong and Kenya) also suggest that hospital readmission rates may be higher in younger than older adults. ${ }^{17}$

Most systematic reviews of predictors for hospital readmission have not highlighted age as a predominate factor. ${ }^{1819}$ Rather, the reviews focus more on comorbidities and other characteristics of patients. These assessments have predominately excluded children and young adults from analysis. Therefore, to advance knowledge of the relation between age and hospital readmission, and to contextualize the contribution of age with other characteristics of patients, the specific aims of this study were to assess US national trends across the age continuum in the risk adjusted odds of unplanned hospital readmission following index admissions for all conditions, accounting for number of chronic conditions, payer, and other characteristics, and to distinguish which US patients admitted to hospital have the highest odds of readmission.

\section{Methods}

\section{Study design and setting}

This was a retrospective cohort analysis of the 2013 Agency for Healthcare Research and Quality's Nationwide Readmissions Database (NRD), a database of hospital inpatient stays for patients of all ages and for all payers. The NRD is drawn from the Agency for Healthcare Research and Quality's state inpatient databases from 21 states with a variety of sizes and population densities. ${ }^{20}$ It contains verified patient identifiers to track an individual across hospital admissions within and across a state's hospitals. The database contains 14.3 million hospital admissions from 2006 hospitals, weighted to represent 35.6 million total admissions nationwide.

\section{Study population}

We identified index admissions for all conditions, including those for observation, between 1 January and 30 November 2013 (to allow for a 30 day readmission window in the full calendar year of data). Guided by methods used by the Centers for Medicare and Medicaid Services, ${ }^{21} 22$ the US federal agency responsible for national measurement, reporting, and policy for hospital readmissions in older people, we excluded index admissions for patients who died or were transferred to another acute care hospital. However, because the intent of this study was to assess hospital readmissions in a broad context for the entire US population, we included patient populations (for example, newborns, children, and people with cancer and mental health conditions) that are typically excluded when reporting US hospital level readmission rates using Centers for Medicare and Medicaid Services methods. ${ }^{2122}$ Readmission policies for adults in other countries do not exempt specific patient groups. ${ }^{1}$ We categorized and analyzed all index admissions and presented them by patient's age at admission, starting with infants (age 0 years) and then progressing in one year epochs through age 90 years or over (the predetermined oldest age category in the database).

\section{Main outcome measure}

The main outcome measure was 30 day, unplanned, all cause hospital readmission following an index admission. For patients aged 18 years or over on their index admission, we defined readmissions by using Centers for Medicare and Medicaid Services methods. ${ }^{22}$ This method is used across US hospitals for public reporting, comparison, and financial penalties associated with hospital readmissions. ${ }^{22}$ For adult patients, this method excludes planned readmissions using the Agency for Healthcare Research and Quality's clinical classification system, which uses principal ICD-9-CM (international classification of diseases, ninth revision, clinical modification) diagnosis and procedure codes to identify admissions that are considered planned or potentially planned (for example, chemotherapy, labor, and delivery). For patients under 18 years of age, we used the pediatric all-cause readmission measure's definition of planned readmissions, endorsed by the National Quality Forum, which is based on a defined set of ICD-9-CM principal procedure codes. ${ }^{21}$ Applying Centers for Medicare and Medicaid Services and National Quality Forum methods, we counted only one readmission within 30 days of discharge and assessed readmissions themselves as index admissions.

\section{Index admission demographics and clinical characteristics}

We assessed characteristics of the index admissions available in the NRD, including sex, payer (Medicare, Medicaid, private, self pay, no charge, and other), length of stay, type of admission (elective versus emergent), and discharge disposition (routine home; to home with skilled home healthcare; to skilled facility based, post-acute care; and left the hospital against medical advice). Routine home disposition indicates hospital discharge to a patient's home without any additional assistance from skilled nursing or other home care professional providers. Home healthcare disposition indicates discharge to a patient's home with additional care giving, functional, and/or medical assistance provided in the home by a skilled nurse or other health professional (such as a physiotherapist). This skilled assistance might include help with activities of daily living, transfers (for example, wheelchair to bed), administration of drugs and medical equipment (for example, oxygen), and palliative care. Facility based, post-acute care disposition indicates discharge to another medical facility that provides intensive rehabilitation, skilled nursing, and/or palliative care. In the US, patients (especially older people) are more likely to use skilled home health or facility based, postacute care after hospital discharge when they have considerable mobility problems, social challenges, and/or limited family support. 
We also assessed the reason for and severity of illness for each index admission by using $3 \mathrm{MHealth}$ Information System's all patient-refined diagnosis related groups. ${ }^{19}$

${ }^{20}$ There are 314 such groups, organized by medical $(\mathrm{n}=195)$ versus procedural $(\mathrm{n}=119)$ reasons for hospital admission. Each groups maps to a mutually exclusive 25 organ systems (that is, major diagnostic categories). We used the Agency for Healthcare Research and Quality's chronic condition indicator to count the number of patients' chronic conditions and to describe the chronic conditions. ${ }^{23}$ This indicator system defines chronic conditions as those expected to last 12 months or longer and meeting one or both of the following criteria: the condition places limitations on self care, independent living, and social interactions; and/or it results in the need for ongoing intervention with medical products, services, and special equipment. On the basis of the distribution of the number of chronic conditions of patients in the study cohort, we used the categories 0,1 , 2-3, 4-5, and 6 or more chronic conditions.

\section{Statistical analysis}

We used weights developed by Agency for Healthcare Research and Quality, the steward of the NRD, to achieve national estimates of index admissions throughout the US. ${ }^{20}$ The database contains a $100 \%$ complete sample of discharges from its 2006 hospitals. We calculated discharge weights by using post-stratification hospital characteristics (census region, urban-rural location, teaching status, bed size, and hospital control) and patient's sex and age. We determined the target universe of inpatient discharges across all hospitals in the US for each stratum-defined by the characteristics of hospitals and patients listed above-by using the Agency for Healthcare Research and Quality's 47 state inpatient databases, which include $95 \%$ of all US hospital discharges, and American Hospital Association hospital discharge counts for hospitals not reported in the state inpatient databases. Within each stratum, each NRD inpatient admission received a discharge weight that was equal to the total number of total US inpatient discharges it represented.

After the weighting, we summarized the patients' demographic and clinical characteristics. We presented continuous variables with medians and interquartile ranges and categorical variables with frequencies and percentages. In bivariable analysis, we made readmission comparisons within subcategories of a characteristic of an index admission (for example, age) by using $\square^{2}$ tests for categorical variables and Wilcoxon rank sum tests for continuous, non-normally distributed variables.

For multivariable analysis, we derived a logistic regression model to estimate the adjusted odds of readmission across patients' ages, using fixed effects to control for confounding variables known to influence the odds of readmission, including the number of chronic conditions, sex, payer, length of stay, type of index admission (elective versus emergent), reason for index admission (all patient-refined diagnosis related group name), severity of illness (all patient-refined diagnosis related group severity of illness scale: 0 (low) to 4 (high)), discharge disposition, and hospital. ${ }^{10} 1314$

${ }^{19}$ In the model, age was entered in one year epochs. We selected age 45 years as the reference group because of its midpoint location in the age range. We also accounted for each hospital in the model by using the NRD's unique hospital identifier as a fixed effect.

Informed by the multivariable results on age, we derived a second set of logistic regression modelsusing the same cofactors described above-to estimate the adjusted, 30 day readmission rate for each reason for admission, using all patient-refined diagnosis related group name, by age category (for example, age $\geq 65$ years). We used SAS version 9.4 for all analyses. We considered P values below 0.001 to be statistically significant owing to the large sample size.

\section{Patient involvement}

Our previous clinical, research, and policy experiences with patients and families led directly to the development of the study's research question and outcome measures. We leveraged the findings from our previous work on patients' and families' experiences with hospital discharge and readmissions. No patients were involved in the recruitment to or conduct of the study. We will disseminate the study findings with national advocacy programs for patients and families (for example, Family Voices).

\section{Results}

\section{Index admissions}

We included 31729762 index admissions in the analysis. Median age at index admission was 53 (interquartile range 27-71) years. Medicare, private insurance, and Medicaid were the primary payer for $39.2 \% \quad(n=12436258), 30.2 \% \quad(n=9585772)$, and $21.5 \%(n=6814492)$ of the index admissions, respectively (table 1). Seventy nine per cent $(n=24948660)$ of index admissions were for patients with one or more chronic conditions; $69.7 \%$ $(n=22100312)$ were for patents with multiple (two or more) chronic conditions (table 1). From age 30 to 44 years, the percentage of index admissions with multiple chronic conditions increased from 38.2\% $(142266 / 372847)$ to $82.6 \% \quad(214306 / 259431)$ $(\mathrm{P}<0.001)$. By age 45, 56.2\% (144267/256496) of patients admitted to hospital had either four to five chronic conditions $(25.7 \% ; n=66015)$ or six or more chronic conditions (30.5\%; $\mathrm{n}=78252)$.

\section{Age trends in unadjusted rates of hospital readmission}

The 30 day unplanned readmission rate following all US index admissions was $11.6 \%(n=3678018)$. As age at index admission increased from 0 to $90+$ years, the unadjusted 30 day readmission rate increased from $2.4 \%$ (94075/3 843 084) to $15.3 \%$ (155 104/1 011373) $(\mathrm{P}<0.001)$ (fig 1). The largest increase in unadjusted readmission rate (from $7.3 \%(27276 / 372847)$ to $14.2 \%$ (36808/259431); $\mathrm{P}<0.001)$ occurred between ages 30 and 44 years (fig 1 ). 


\begin{tabular}{|c|c|}
\hline Characteristic & Value $(n=31729762)$ \\
\hline Median (IQR) age at admission, years & $53(27-71)$ \\
\hline Male sex & $13416008(42.3)$ \\
\hline \multicolumn{2}{|l|}{ Payer: } \\
\hline Medicare & $12436258(39.2)$ \\
\hline Medicaid & $6814492(21.5)$ \\
\hline Private & $9585772(30.2)$ \\
\hline Self pay & $1488205(4.7)$ \\
\hline No charge & $185238(0.6)$ \\
\hline Other & $1178369(3.7)$ \\
\hline \multicolumn{2}{|l|}{ No of chronic conditions: } \\
\hline 0 & $6781102(21.4)$ \\
\hline 1 & $2848348(9.0)$ \\
\hline $2-3$ & $5447279(17.2)$ \\
\hline $4-5$ & $5900946(18.6)$ \\
\hline$\geq 6$ & 10752087 (33.9) \\
\hline \multicolumn{2}{|l|}{ Type of index admission: } \\
\hline Elective & $6885744(21.7)$ \\
\hline Emergent & $24796482(78.1)$ \\
\hline \multicolumn{2}{|l|}{ Discharge disposition: } \\
\hline Routine & $23455202(73.9)$ \\
\hline Post-acute care & $3981710(12.6)$ \\
\hline Home healthcare & $3918646(12.4)$ \\
\hline Against medical advice & $351351(1.1)$ \\
\hline Unknown & $13405(0.0)$ \\
\hline Median (IQR) length of index admission stay, days & $3(2-5)$ \\
\hline
\end{tabular}

$\mathrm{IQR}=$ interquartile range.
$2199708 / 23455202)$ than patients discharged to home health $(17.1 \%$; $668457 / 3918646)$ or postacute facility care $(18.0 \% ; 718632 / 3981710)$ or patients who left the hospital against medical advice (25.4\%; 89192/351351). Payer and discharge disposition remained significantly associated $(\mathrm{P}<0.001)$ with hospital readmission in multivariable analysis (table 2). For discharge disposition, patients who left against medical advice had the highest odds of readmission compared with patients with routine discharge to home (odds ratio 2.11, 2.10 to 2.13).

Number of chronic conditions was also significantly associated with hospital readmission. In bivariable analysis, readmission rates increased from $2.7 \%$ (181 807/6 781 102) to $18.6 \%$ (1 999 481/10 752 087) as the number of chronic conditions increased from none to six or more $(\mathrm{P}<0.001)$. In multivariable analysis, multiple chronic conditions-of all characteristicswere associated with the highest odds of readmission (for example, odds ratio 3.67 (3.64 to 3.69) for $\geq 6 v 0$ chronic conditions) (table 2).

After we had accounted for patients' demographic and clinical characteristics, the odds of readmission varied significantly across hospitals. The adjusted odds ratios for hospitals ranged from 0.03 (0.00 to 0.16 ) to 10.22 (8.62 to 12.13 ).

\section{Age trends in the adjusted odds of hospital readmission}

Referenced by patients aged 45 years, the range of risk adjusted odds ratios for readmission decreased for children from age 0 to 6 years (0.87 (0.85 to 0.88) to 0.58 (0.56 to 0.60$)$ ) (fig 2). A substantial increase in the odds of readmission occurred between ages 16 and 20 years ( 0.70 (0.68 to 0.71 ) to 1.04 (1.02 to 1.06)). Patients aged 21-44 years had similar odds of readmission (odds ratio range 1.02 (1.00 to 1.03) to 1.12 (1.10 to 1.14)), compared with patients aged 45 years. As patients' age increased from 46 to 64 years, the odds of readmission decreased from 1.02 (1.00 to 1.04 ) to 0.91 (0.90 to 0.93$)$. At age 65 , the odds of readmission decreased to 0.78 (0.77 to 0.79) and stayed relatively constant through age $90+$ years (range 0.67 (0.66 to 0.67 ) to 0.78 (0.76 to 0.79$)$ ) (fig 2).

\section{Variation in adjusted readmission rates by reasons for index admission}

After adjusting for patients' clinical and demographic characteristics, we observed significant $(\mathrm{P}<0.001)$ variation in readmission rates across the reasons for index admission (fig 3) Among children, young adults, and middle aged adults, mental health was one of the most common reasons for index admissions that had a high adjusted readmission rate ( $\geq 75$ th centile). Specific mental health conditions included bipolar disorder, major depression, schizophrenia, and substance misuse/dependence (for example, alcohol and opioids). Among older patients ( $\geq 65$ years), septicemia and kidney/urinary tract infections were the most common reasons for index admissions that had a high adjusted readmission rate (fig 3).
Fig 1 | Age trends in unadjusted, 30 day, unplanned readmission rates per 100 index hospital admissions 


\begin{tabular}{|c|c|c|}
\hline \multirow[b]{2}{*}{ Characteristic } & \multicolumn{2}{|c|}{30 day unplanned hospital readmission } \\
\hline & No $(\% *)$ & $\begin{array}{l}\text { Adjusted odds ratio } \\
\text { for readmission }(95 \% \mathrm{Cl}) \dagger\end{array}$ \\
\hline \multicolumn{3}{|l|}{ Sex: } \\
\hline Female & $1929809 / 18313755(10.5)$ & Reference \\
\hline Male & $1748209 / 13416008$ (13.0) & $1.02(1.02$ to 1.02$)$ \\
\hline \multicolumn{3}{|l|}{ Payer: } \\
\hline Medicare & $2042383 / 12436258$ (16.4) & $1.50(1.50$ to 1.51$)$ \\
\hline Medicaid & $690093 / 6814492(10.1)$ & $1.50(1.50$ to 1.51$)$ \\
\hline Private & $666738 / 9585772(7.0)$ & Reference \\
\hline Self pay & $143399 / 1488205(9.6)$ & $0.95(0.95$ to 0.96$)$ \\
\hline No charge & $21839 / 185238(11.8)$ & $1.00(0.99$ to 1.02$)$ \\
\hline Other & $109376 / 1178369(9.3)$ & 1.07 (1.06 to 1.08$)$ \\
\hline \multicolumn{3}{|c|}{$\begin{array}{l}\text { No of organ systems affected by } \\
\text { chronic conditions: }\end{array}$} \\
\hline 0 & $181807 / 6781102(2.7)$ & Reference \\
\hline 1 & $164583 / 2848348(5.8)$ & $1.44(1.43$ to 1.45$)$ \\
\hline $2-3$ & $547262 / 5447279(10.0)$ & 2.08 (2.07 to 2.10$)$ \\
\hline $4-5$ & $784885 / 5900946(13.3)$ & $2.64(2.62$ to 2.66$)$ \\
\hline$\geq 6$ & $1999481 / 10752087$ (18.6) & 3.67 (3.64 to 3.69 ) \\
\hline Severity of illness $\neq$ & NA & $1.02(1.02$ to 1.02$)$ \\
\hline Length of stay & NA & 1.01 (1.01 to 1.01$)$ \\
\hline \multicolumn{3}{|l|}{ Type of index admission: } \\
\hline Elective & $514893 / 6885744(7.5)$ & Reference \\
\hline Emergent & $3157578 / 24796482(12.7)$ & $1.44(1.43$ to 1.44$)$ \\
\hline \multicolumn{3}{|l|}{ Discharge disposition: } \\
\hline Routine & $2199708 / 23455202(9.4)$ & Reference \\
\hline Post-acute facility care & $718632 / 3981710(18.0)$ & $1.37(1.36$ to 1.37$)$ \\
\hline Home healthcare & $668457 / 3918646(17.1)$ & $1.41(1.40$ to 1.41$)$ \\
\hline Against medical advice & $89192 / 351351(25.4)$ & 2.11 (2.10 to 2.13$)$ \\
\hline Unknown & $653 / 13405(4.9)$ & $0.30(0.28$ to 0.33$)$ \\
\hline
\end{tabular}

NA=not applicable.

*Percentages are readmission rates.

† Multivariable odds ratios derived using logistic regression with fixed effects for all characteristics shown in table

as well as age (in 1 year increments), controlled for clustering of data by hospital.

¥Measured with 3M Health Information System’s all patient-refined diagnosis related groups.

\section{Discussion}

The main findings from this US study enhance knowledge about hospital readmissions. As regards age-the primary focus of the work-the adjusted odds of 30 day, unplanned hospital readmission increased substantially from adolescence through

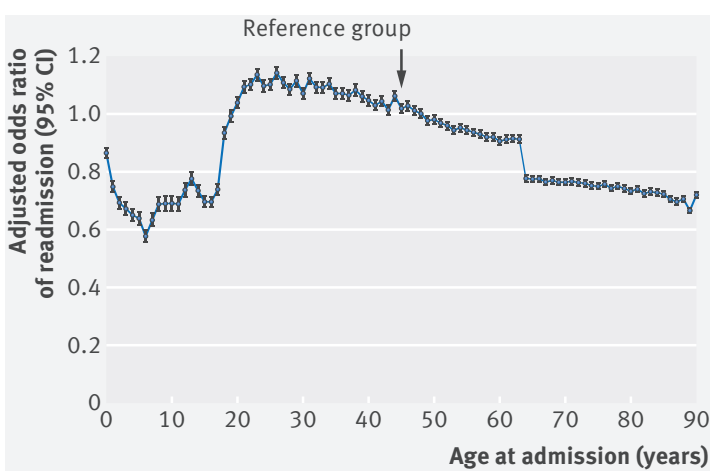

Fig 2 | Age trends in adjusted likelihood of 30 day, unplanned hospital readmission. Reference group=age 45 years, midpoint in range of age. Multivariable odds ratios were adjusted for number of chronic conditions, sex, payer, reason for and severity of admission, type of admission (elective $v$ emergent), length of stay, and discharge disposition young adulthood, where the highest odds of readmission were observed across all ages. Mental health was a prominent reason for index admissions with high readmission rates observed in children as well as young and middle aged adults. A substantial decrease in the odds of readmission occurred at age 65 years. In addition to age, other important risk factors emerged that had stronger associations with hospital readmission. For example, leaving the hospital against medical advice and having multiple chronic conditions were associated the highest adjusted odds of readmission of all characteristics of patients.

\section{Comparison with other studies}

Additional investigation is needed to explain the reasons for the substantial decrease in the odds of hospital readmission from age 64 to 65 years for US patients. This finding was not reported in a previous US readmission study using the same dataset that stratified people admitted to hospital into broad categories at that age juncture (for example, age 45-64 $V \geq 65$ years). ${ }^{24}$ At age 65 years, US adults enroll in Medicare, the federal health insurance plan exclusively for people aged 65 and older. ${ }^{25}$ Hospital health services in the US typically do not change between ages 64 and 65 years; acute care hospitals do not restrict US patients aged 65 years or over to certain hospital units or providers. In contrast, certain US federal, outpatient based readmission interventions (such as the Community-based Care Transitions program) create partnerships between hospital and community providers to reduce readmissions exclusively for Medicare beneficiaries aged 65 years or over. ${ }^{26}{ }^{27}$ Moreover, most US readmission policies with financial penalties target people aged 65 years and over. ${ }^{2}$ In contrast, readmission policies for adults in other countries, including the UK, Denmark, and Germany, do not exempt specific ages. ${ }^{1}$ Although most US hospitals strive to provide high quality discharge care for all patients, it is possible that US national readmission reporting and reduction policies contributed to enhanced discharge planning for people aged 65 years or over in some hospitals. ${ }^{28-31}$

This study highlights the importance of mental health conditions-including psychiatric and substance misuse disorders-in readmissions. Previous US reports and studies using the same or similar data from the Agency for Healthcare Research and Quality show high unadjusted readmission rates for adults with mental health conditions. ${ }^{524}$ Our study upholds those mental health findings by using risk adjustment methods and also extends them to children and adolescents. In contrast to other countries, patients admitted to hospital with mental health conditions are typically excluded from readmission measurement and policy in the US. ${ }^{1}$ Nevertheless, our findings are similar to those in the UK, where children with psychiatric and substance misuse disorders have high readmission rates. ${ }^{32}$ Moreover, systematic reviews of studies across the globe report increasing overdose related hospital admissions, with peaks at the current time. ${ }^{33}$ The 


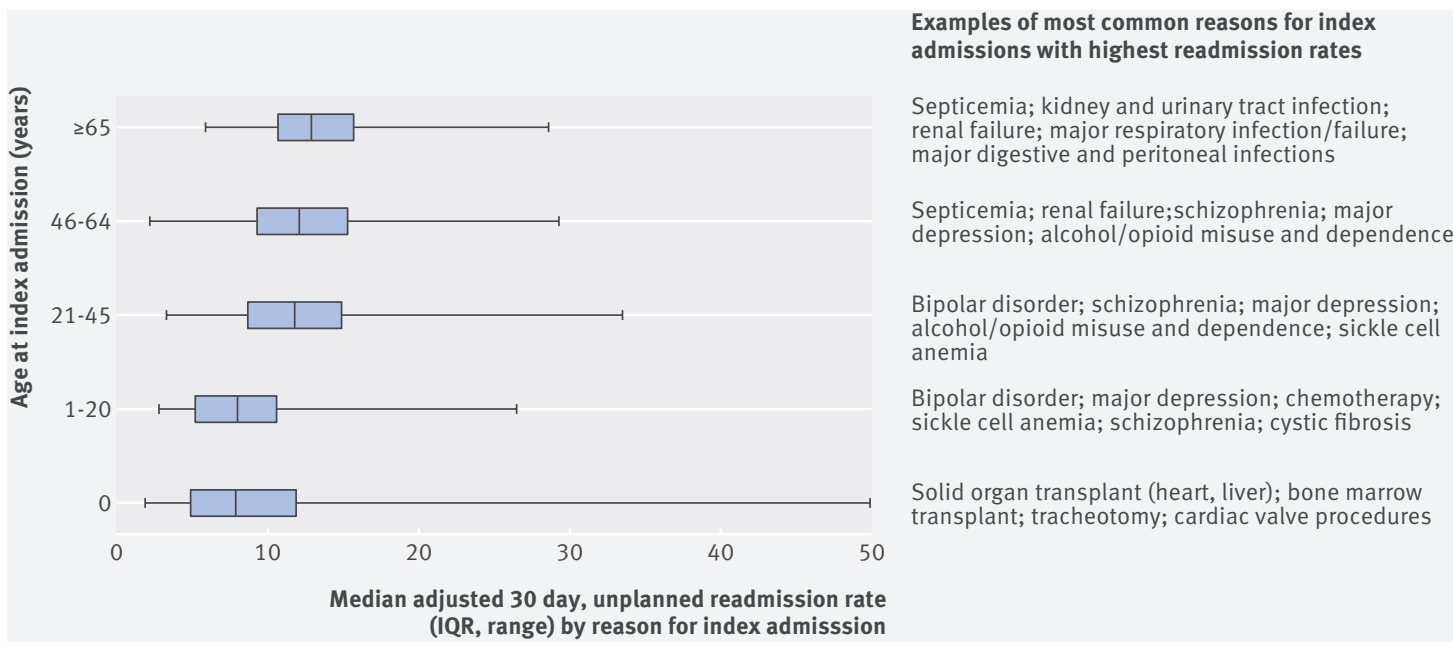

Fig 3 | Variation in 30 day, unplanned hospital readmission by reason for index admission. $X$ axis shows distributions of 30 day readmission rates across 314 reasons for index admission, distinguished with all patient-refined diagnosis related groups categories, for each age group. Rates were adjusted for sex, number of chronic conditions, severity of illness, type of admission (elective $v$ emergent), length of stay, and discharge disposition. Midline of box is median rate by all patient-refined diagnosis related groups. Left and right borders of box are 25th and 75th centiles of rate by groups. Whiskers are minimum and maximum rates. Examples of most common reasons for index admissions within highest quarter ( $\geq 75$ th centile) of readmission rates are listed for each age category. IQR=interquartile range

World Health Organization's 2014 Mental Health Atlas reports a wide gap in mental health services available between higher and lower income countries, especially as regards availability of day treatment and community residential facilities. ${ }^{34}$ Even in higher income countries, including the US, acute care hospitals struggle to identify sufficient outpatient and community resources that will optimize the adherence to treatment, social stability, and emotional health of people discharged with a mental health problem..$^{35-39}$

The increase in odds of hospital readmission from adolescence through young adulthood in this study warrants further exploration. The methods of previous national US readmission studies precluded discovery of this finding by excluding children and adolescents and by not analyzing age in one year epochs. ${ }^{5} 24$ The International and Interdisciplinary Health Care Transition Research Consortium, with representatives from all continents, prioritizes unnecessary hospital admission as a key outcome to avoid for young people during their transition to adulthood. ${ }^{40}$ The health and healthcare experiences during transition from childhood to adulthood might help to explain the increasing odds of readmission observed during that time. Many children with complex chronic conditions (such as diabetes or sickle cell anemia) experience a progressive worsening in severity of illness, often with the development of comorbidities, as they move into adulthood. ${ }^{41-43}$ Unfortunately, this worsening coincides with increasing self management responsibilities for young people in the setting of healthcare challengesreported worldwide-that they experience, including discontinuity of and difficulty accessing health services, as well as lack of care coordination during transfer of care from pediatric to adult healthcare providers. ${ }^{44-50}$ Emerging evidence suggests that some pediatric clinicians are not sufficiently preparing children for these experiences. ${ }^{51}$ During children's adolescent years, those pediatric clinicians are not promoting or offering enough health autonomy and responsibility to the children. Underuse of health services and high rates of unmet healthcare needs are reported for young adults with disabling, chronic health conditions. ${ }^{52}$ Further investigation is needed to determine whether these experiences are contributing to the higher odds of readmission in young adults, especially those with multiple chronic conditions.

The finding of higher odds of readmission following index admissions for all conditions in younger compared with older people observed in our current study complements previous literature. Similar or higher adjusted readmission rates have been reported for younger versus older US adults following index admissions for specific conditions, including heart failure, acute myocardial dysfunction, and pneumonia. ${ }^{16}$ Hospital readmission studies from Asia, Africa, and other continents also report higher rates of readmission in younger versus older adults. ${ }^{17}$ This finding might be partially explained by differences between younger and older people in the type, pathophysiology, and associated healthcare needs of chronic conditions that they experience. For example, for some patients, the onset of heart failure in younger versus older people might indicate a higher severity and complexity of illness. ${ }^{1653}$ Although our risk adjustment methods included discharge disposition, the enhanced post-discharge community supports (for example, rehabilitation and skilled nursing facility care) available to help mitigate readmission risk for older adults might not be as accessible for younger people. ${ }^{54}$

Post-discharge death could have also influenced the findings of readmission for younger versus older 
adults in our study. Lacking information on deaths outside of the hospital, we could not distinguish which patients died after discharge, thereby negating their risk of readmission. Previous studies on index admissions for specific conditions report inconsistent findings on the competing risks of post-discharge death and readmission; some studies report an inverse correlation, whereas others report no correlation or a positive one. ${ }^{55-57}$ Competing risk of post-discharge death is not assessed when publicly reporting and comparing US hospitals' performance on readmissions. Certainly, the risk of death (at any time) increases with age and likely influences, to some degree, the odds of readmission observed for older people in our study. Countries beyond the US have prioritized the focus of readmissions on younger patients because they are more likely to survive, so they particularly warrant the investment in high quality discharge care. ${ }^{17}$ Nevertheless, the competing risk of post-discharge death should not permit leniency of hospital and outpatient follow-up clinicians in delivering high quality discharge care to people of advanced age.

Beyond age, our study underscores the effect of additional risk factors for readmission, including multiple chronic conditions. In multivariable analysis, the effect of multiple chronic conditions on the likelihood of readmission substantially overshadowed other characteristics of patients, including age. People with multiple chronic conditions have fragile health status, concurrent risk of exacerbation for each chronic condition, complicated discharge planning, and enhanced need for coordination of post-discharge care. ${ }^{58-61}$ Although the number of chronic conditions is included, to some degree, for risk adjustment in some US readmission measures and policies, ${ }^{22} 6263$ greater population based emphasis on optimizing the discharge care, follow-up care, and overall health of people with multiple chronic conditions is warranted. Although older people in our study had the highest prevalence of multiple chronic conditions, assessing the effect of scaling effective care transition programs for them to younger people may be particularly important.

Leaving against medical advice was another risk factor with a strong association with hospital readmission. Previous studies of index admissions for all conditions in Canada and Australia also report higher odds of hospital readmission in patients who leave against medical advice. ${ }^{6465}$ Clinical frameworks of hospital discharge care highlight the importance of readiness for discharge as a key driver of health after leaving the hospital. ${ }^{66}$ Being unprepared for discharge from hospital has been associated with higher odds of readmission in both children and adults. ${ }^{61} 67$ Nevertheless, some patients have family, employment, financial, or other problems that necessitate their wish to leave before their hospital clinicians consider them ready for discharge. ${ }^{68}$ Others have a history of mental health disorders and/or substance misuse that influences their decision to leave. ${ }^{68}$ Better efforts to disclose and mitigate the health risk taken by people who leave the hospital against medical advice are needed, especially as these people are excluded from most US studies on hospital readmission as well as US readmission policies. ${ }^{1}$

\section{Limitations of study}

This study has several limitations. All findings are national estimates generated from the Nationwide Readmissions Database sample; the accuracy of the estimates and their associated variances depend on the weighting methods developed by the Agency for Healthcare Research and Quality. Although we used indicators for the reason for and severity of admission, number of chronic conditions, and other characteristics of patients in the analyses, differences in the case mix of index admissions across the age spectrum could remain. The NRD does not include readmissions to hospitals in a different state, which could have resulted in undercounting of readmissions. The database is not positioned to assess social determinants of health, outpatient care, or other factors that influence patients' health and healthcare during transitions from hospital to home.

Although we accounted for discharge disposition to post-acute and home healthcare-health services typically used by patients with mobility and other functional impairments as well as limitations in family and social supports-the NRD does not contain direct information on those characteristics of patients and families. We excluded planned readmissions from measurement, but validation of the methods used to distinguish planned readmissions (used by the US Centers for Medicare and Medicaid Services and the National Quality Forum) beyond expert opinion is not forthcoming in previous literature or reports. In a post-hoc analysis, we did statistical analysis for all cause readmissions including those for planned readmissions; aside from higher unadjusted readmission rates, the main study findings did not change. The NRD administrative data used in this study are not designed to distinguish preventable readmissions.

\section{Conclusions and policy implications}

Despite the above limitations, the main findings from this US study show important trends in the adjusted odds of hospital readmission across the entire age continuum, highlight certain index admissions that are associated with high adjusted readmission rates for both children and adults, and distinguish several risk factors beyond age that have strong associations with hospital readmission. When contextualized with findings from previous readmission studies worldwide, certain patient populations of importance emerge for consideration in future research, quality improvement, and policy efforts. These populations include children transitioning to adulthood, children and younger adults with mental health and substance misuse disorders, and people of all ages with multiple chronic conditions. Future efforts in the US, in particular, may also benefit from exploring how the current 
US readmission policies and patterns of insurance coverage could have contributed to the abrupt decrease in the odds of readmission at age 65 years. Although people aged 65 years and older in the US and beyond may continue to account for a disproportionate share of all hospital readmissions, increased attention to other at risk, vulnerable patients may be necessary to optimize hospital discharge and follow-up care across the age continuum.

Contributors: All authors made substantial contributions to the conception or design of the work. MH acquired and analyzed the data. All authors were involved in interpreting data and in drafting the manuscript and revising it critically for important intellectual content. All authors gave final approval of the version to be published and have agreed to be accountable for all aspects of the work in ensuring that questions related to the accuracy or integrity of any part of the work are appropriately investigated and resolved. JGB is the guarantor. Funding: JGB and MH were supported by the Agency for Healthcare Research and Quality (R21 HS023092-01). JGB, KB, and MRO were supported by the Lucile Packard Foundation for Children's Health. The funders were not involved in the study design; in the collection, analysis, and interpretation of data; in the writing of the report; or in the decision to submit the article for publication. The researchers conducted the work independently of the funders.

Competing interests: All authors have completed the ICMJE uniform disclosure form at www.icmje.org/coi_disclosure.pdf (available on request from the corresponding author) and declare: no support from any organization for the submitted work other than that described above; no financial relationships with any organizations that might have an interest in the submitted work in the previous three years; no other relationships or activities that could appear to have influenced the submitted work

Ethical approval: This study was not considered human subject research by the Institutional Review Board at Vanderbilt University Medical Center, and ethics committee approval was therefore not required.

Transparency: The lead author (JGB) affirms that the manuscript is an honest, accurate, and transparent account of the study being reported; that no important aspects of the study have been omitted; and that any discrepancies from the study as planned (and, if relevant, registered) have been explained.

Data sharing: No additional data available.

This is an Open Access article distributed in accordance with the Creative Commons Attribution Non Commercial (CC BY-NC 4.0) license, which permits others to distribute, remix, adapt, build upon this work non-commercially, and license their derivative works on different terms, provided the original work is properly cited and the use is noncommercial. See: http://creativecommons.org/licenses/by-nc/4.0/.

1 Kristensen SR, Bech M, Quentin W. A roadmap for comparing readmission policies with application to Denmark, England, Germany and the United States. Health Policy 2015;119:264-73. 10.1016/ j.healthpol.2014.12.009

2 Jencks SF, Williams MV, Coleman EA. Rehospitalizations among patients in the Medicare fee-for-service program. N Engl J Med 2009;360:1418-28. 10.1056/NEJMsa0803563

3 Kassler WJ, Tomoyasu N, Conway PH. Beyond a traditional payer--CMS's role in improving population health. N Engl J Med 2015;372:109-11. 10.1056/NEJMp1406838

4 Jiang HJ, Barrett ML, Sheng M. Characteristics of hospital stays for nonelderly Medicaid super-utilizers, 2012. HCUP statistical brief \#184. Agency for Healthcare Research and Quality, 2014.

5 Jiang HJ, Wier LM. All-cause hospital readmissions among non-elderly Medicaid patients, 2007. HCUP statistical brief \#89. Agency for Healthcare Research and Quality, 2010.

6 Podulka J, Barrett M, Jiang HJ, Steiner C. 30-day readmissions following hospitalizations for chronic vs. acute conditions, 2008. HCUP statistical brief \#127. Agency for Healthcare Research and Quality, 2012

7 Barrett ML, Wier LM, Jiang HJ, Steiner CA. All-cause readmissions by payer and age, 2009-2013. HCUP statistical brief \#199. Agency for Healthcare Research and Quality, 2014.

8 Wier LM, Barrett M, Steiner C, Jiang HJ. All-cause readmissions by payer and age, 2008: HCUP statistical brief\#115. Agency for Healthcare Research and Quality, 2011.
9 Wijlaars LP, Hardelid P, Woodman J, Allister J, Cheung R, Gilbert R. Who comes back with what: a retrospective database study on reasons for emergency readmission to hospital in children and young people in England. Arch Dis Child 2016;101:714-8. 10.1136/ archdischild-2015-309290

10 Berry JG, Toomey SL, Zaslavsky AM, et al. Pediatric readmission prevalence and variability across hospitals. JAMA 2013;309:372-80 10.1001/jama.2012.188351

11 Sambamoorthi U, Tan X. Deb A. Multiple chronic conditions and healthcare costs among adults. Expert Rev Pharmacoecon Outcomes Res 2015;15:823-32. 10.1586/14737167.2015.1091730

12 Vogeli C, Shields AE, Lee TA, et al. Multiple chronic conditions: prevalence, health consequences, and implications for quality, care management, and costs. J Gen Intern Med 2007;22(Suppl 3):391-5. 10.1007/s11606-007-0322-1

13 Fuller RL, Atkinson G, McCullough EC, Hughes JS. Hospital readmission rates: the impacts of age, payer, and mental health diagnoses. J Ambul Care Manage 2013;36:147-55. 10.1097/ JAC.0b013e3182866c1c

14 Berry JG, Hall DE, Kuo DZ, et al. Hospital utilization and characteristics of patients experiencing recurrent readmissions within children's hospitals. JAMA 2011;305:682-90. 10.1001/jama.2011.122

15 Chang DW, Tseng CH, Shapiro MF. Rehospitalizations Following Sepsis: Common and Costly. Crit Care Med 2015;43:2085-93. 10.1097/CCM.0000000000001159

16 Ranasinghe I, Wang Y, Dharmarajan K, Hsieh AF, Bernheim SM, Krumholz HM. Readmissions after hospitalization for heart failure, acute myocardial infarction, or pneumonia among young and middle-aged adults: a retrospective observational cohort study. PLoS Med 2014:11:e1001737. 10.1371/journal.pmed.1001737

17 Amoah D, Mwanri L. Determinants of hospital readmissions of medical conditions in developing countries. Austin Journal of Public Health and Epidemiology 2016;3(5).

18 Zhou H, Della PR, Roberts P, Goh L, Dhaliwal SS. Utility of models to predict 28-day or 30-day unplanned hospital readmissions: an updated systematic review. BMJ Open 2016;6:e011060. 10.1136/ bmjopen-2016-011060

19 Kansagara D, Englander H, Salanitro A, et al. Risk prediction models for hospital readmission: a systematic review. JAMA 2011;306: 1688-98. 10.1001/jama.2011.1515

20 Agency for Healthcare Research and Quality Healthcare Cost and Utilization Project (HCUP). Introduction to the HCUP Nationwide Readmissions Database (NRD), 2013. 2015. https://www.hcup-us. ahrq.gov/db/nation/nrd/NRD_Introduction_2013.jsp.

21 Schuster MA. Pediatric all-condition readmission measure. Agency for Healthcare Research and Quality, 2014.

22 van den Bosch WF, Kelder JC, Wagner C. Predicting hospital mortality among frequently readmitted patients: HSMR biased by readmission BMC Health Serv Res 2011:11:57. 10.1186/1472-6963-11-57

23 Healthcare Cost and Utilization Project (HCUP). Chronic conditions indicator for ICD-9-CM. 2016. http://www.hcup-us.ahrq.gov/ toolssoftware/chronic/chronic.jsp.

24 Strom JB, Kramer DB, Wang Y, et al. Short-term rehospitalization across the spectrum of age and insurance types in the United States. PLoS One 2017;12:e0180767. 10.1371/journal.pone.0180767

25 Huffman KF, Upchurch G. The Health of Older Americans: A Primer on Medicare and a Local Perspective. J Am Geriatr Soc 2018;66:25-32. 10.1111/jgs.15227

26 Hung D, Truong Q, Yakir M, Nicosia F. Hospital-Community Partnerships to Aid Transitions for Older Adults: Applying the Care Transitions Framework. J Nurs Care Qual 2017. 10.1097/ NCQ.0000000000000294

27 Jenq GY, Doyle MM, Belton BM, Herrin J, Horwitz LI. QuasiExperimental Evaluation of the Effectiveness of a Large-Scale Readmission Reduction Program. JAMA Intern Med 2016;176 681-90. 10.1001/jamainternmed.2016.0833

28 Gerhardt G, Yemane A, Hickman P, Oelschlaeger A, Rollins E, Brennan N. Medicare readmission rates showed meaningful decline in 2012. Medicare Medicaid Res Rev 2013;3:mmrr.003.02.b01. 10.5600/mmrr.003.02.b01

29 Suter LG, Li SX, Grady JN, et al. National patterns of riskstandardized mortality and readmission after hospitalization for acute myocardial infarction, heart failure, and pneumonia: update on publicly reported outcomes measures based on the 2013 release. J Gen Intern Med 2014;29:1333-40. 10.1007/s11606014-2862-5

30 Zuckerman RB, Sheingold SH, Orav EJ, Ruhter J, Epstein AM. Readmissions, Observation, and the Hospital Readmissions Reduction Program. N Engl J Med 2016;374:1543-51. 10.1056/ NEIMsa1513024

31 Joynt KE, Figueroa JE, Oray J, Jha AK. Opinions on the Hospital Readmission Reduction Program: results of a national survey of hospital leaders. Am J Manag Care 2016;22:e287-94. 
32 Hoy AR. Which young people in England are most at risk of an alcohol-related revolving-door readmission career? BMC Public Health 2017;17:185. 10.1186/s12889-016-3891-2

33 Martins SS, Sampson L, Cerdá M, Galea S. Worldwide Prevalence and Trends in Unintentional Drug Overdose: A Systematic Review of the Literature. Am J Public Health 2015;105:e29-49. 10.2105 AJPH.2015.302843

34 Lora A, Hanna F, Chisholm D. Mental health service availability and delivery at the global level: an analysis by countries' income level from WHO's Mental Health Atlas 2014. Epidemiol Psychiatr Sci 2017;1-12. 10.1017/S2045796017000075

35 Bardach NS, Coker TR, Zima BT, et al. Common and costly hospitalizations for pediatric mental health disorders. Pediatrics 2014;133:602-9. 10.1542/peds.2013-3165

36 Torio CM, Encinosa W, Berdahl T, McCormick MC, Simpson LA. Annual report on health care for children and youth in the United States: national estimates of cost, utilization and expenditures for children with mental health conditions. Acad Pediatr 2015;15:19-35. 10.1016/j.acap.2014.07.007

37 Freeland KN, Cogdill BR, Ross CA, et al. Adherence to evidence-based treatment guidelines for bipolar depression in an inpatient setting. Am J Health Syst Pharm 2015;72(Suppl 3):S156-61. 10.2146/ sp150023

38 Hamilton JE, Passos IC, de Azevedo Cardoso T, et al. Predictors of psychiatric readmission among patients with bipolar disorder at an academic safety-net hospital. Aust N Z J Psychiatry 2016;50:584-93. 10.1177/0004867415605171

39 Lafeuille MH, Dean J, Carter V, et al. Systematic review of long-acting injectables versus oral atypical antipsychotics on hospitalization in schizophrenia. Curr Med Res Opin 2014;30:1643-55. 10.1185/03007995.2014.915211

40 Fair C, Cuttance J, Sharma N, et al, International and Interdisciplinary Health Care Transition Research Consortium. International and Interdisciplinary Identification of Health Care Transition Outcomes. JAMA Pediatr 2016;170:205-11. 10.1001/ jamapediatrics.2015.3168

41 Quinn CT. Sickle cell disease in childhood: from newborn screening through transition to adult medical care. Pediatr Clin North Am 2013;60:1363-81. 10.1016/j.pcl.2013.09.006

42 Sawicki GS, Tiddens H. Managing treatment complexity in cystic fibrosis: challenges and opportunities. Pediatr Pulmonol 2012; 47:523-33. 10.1002/ppul.22546

43 Ferro MA, Gorter JW, Boyle MH. Trajectories of depressive symptoms during the transition to young adulthood: the role of chronic illness. Affect Disord 2015;174:594-601. 10.1016/j.jad.2014.12.014

44 Bibbins-Domingo K, Coxson P, Pletcher MJ, Lightwood J, Goldman L. Adolescent overweight and future adult coronary heart disease. N Engl J Med 2007;357:2371-9. 10.1056/ NEJMsa073166

45 Goossens E, Bovijn L, Gewillig M, Budts W, Moons P. Predictors of Care Gaps in Adolescents With Complex Chronic Condition Transitioning to Adulthood. Pediatrics 2016;137:e20152413. 10.1542/peds.2015-2413

46 Adams-Graves P, Bronte-Jordan L. Recent treatment guidelines for managing adult patients with sickle cell disease: challenges in access to care, social issues, and adherence. Expert Rev Hematol 2016;9:541-52. 10.1080/17474086.2016.1180242

47 Bailey H, Cruz MLS, Songtaweesin WN, Puthanakit T. Adolescents with HIV and transition to adult care in the Caribbean, Central America and South America, Eastern Europe and Asia and Pacific regions. J Int AIDS Soc 2017;20(Suppl 3):21475. 10.7448/ IAS.20.4.21475

48 Treuer T, Chan KLP, Kim BN, et al. Lost in transition: A review of the unmet need of patients with attention deficit/hyperactivity disorder transitioning to adulthood. Asia Pac Psychiatry 2017;9. 10.1111/ appy. 12254
49 Honda M, lijima K, Ishikura K, Kaneko K. The problem of transition from pediatric to adult healthcare in patients with steroid-sensitive nephrotic syndrome (SSNS): a survey of the experts. Clin Exp Nephrol 2014;18:939-43. 10.1007/s10157-014-0941-x

50 Bower WF, Christie D, DeGennaro M, et al. The transition of young adults with lifelong urological needs from pediatric to adult services: An international children's continence society position statement. Neurourol Urodyn 2017;36:811-9. 10.1002/nau.23039

51 American Academy of Pediatrics, American Academy of Family Physicians, American College of Physicians-American Society of Internal Medicine. A consensus statement on health care transitions for young adults with special health care needs. Pediatrics 2002:110:1304-6.

52 Callahan ST, Cooper WO. Access to health care for young adults with disabling chronic conditions. Arch Pediatr Adolesc Med 2006;160:178-82. 10.1001/archpedi.160.2.178

53 Greutmann M, Tobler D, Kovacs AH, et al. Increasing mortality burden among adults with complex congenital heart disease. Congenit Heart Dis 2015;10:117-27. 10.1111/chd.12201

54 Berry JG, Hall M, Dumas H, et al. Pediatric Hospital Discharges to Home Health and Postacute Facility Care: A National Study. JAMA Pediatr 2016;170:326-33. 10.1001/jamapediatrics.2015.4836

55 Fonarow GC, Konstam MA, Yancy CW. The Hospital Readmission Reduction Program Is Associated With Fewer Readmissions, More Deaths: Time to Reconsider. J Am Coll Cardiol 2017;70:1931-4. 10.1016/j.jacc. 2017.08 .046

56 Dharmarajan K, Wang Y, Lin Z, et al. Association of Changing Hospital Readmission Rates With Mortality Rates After Hospital Discharge. JAMA 2017;318:270-8. 10.1001/jama.2017.8444

57 Eapen ZJ, Reed SD, Li Y, et al. Do countries or hospitals with longer hospital stays for acute heart failure have lower readmission rates?: Findings from ASCEND-HF. Circ Heart Fail 2013;6:727-32. 10.1161/ CIRCHEARTFAILURE.112.000265

58 Tinetti ME, Bogardus ST Jr, Agostini JV. Potential pitfalls of diseasespecific guidelines for patients with multiple conditions. $N$ Engl Med 2004:351:2870-4. 10.1056/NEJMsb042458

59 Berry JG, Hall M, Dumas H, et al. Pediatric Hospital Discharges to Home Health and Postacute Facility Care: A National Study. JAMA Pediatr 2016;170:326-33. 10.1001/ jamapediatrics.2015.4836

60 Parekh AK, Kronick R, Tavenner M. Optimizing health for persons with multiple chronic conditions. JAMA 2014;312:1199-200. 10.1001/jama.2014.10181

61 Berry JG, Ziniel SI, Freeman L, et al. Hospital readmission and parent perceptions of their child's hospital discharge. Int I Qual Health Care 2013:25:573-81. 10.1093/intahc/mzt051

62 National Quality Forum. Endorsement summary: all-cause readmissions. National Quality Forum, 2012

63 National Quality Forum. All-cause admissions and readmissions measures: final report. National Quality Forum, 2015.

64 Garland A, Ramsey CD, Fransoo R, et al. Rates of readmission and death associated with leaving hospital against medical advice: a populationbased study. CMA/ 2013:185:1207-14. 10.1503/cmaj.130029

65 Considine J, Fox K, Plunkett D, Mecner M, O'Reilly M, Darzins P. Factors associated with unplanned readmissions in a major Australian health service. Aust Health Rev 2017. 10.1071/AH16287

66 Berry JG, Blaine K, Rogers J, et al. A framework of pediatric hospital discharge care informed by legislation, research, and practice. JAMA Pediatr 2014;168:955-62, quiz 965-6. 10.1001/ amapediatrics.2014.891

67 Coleman EA, Mahoney E, Parry C. Assessing the quality of preparation for posthospital care from the patient's perspective: the care transitions measure. Med Care 2005:43:246-55. 10.1097/00005650-200503000-00007

68 Alfandre DJ. "I'm going home": discharges against medical advice. Mayo Clin Proc 2009:84:25 5-60. 10.4065/84.3.25 